\title{
Relative Humidity Sensor Based on Microfiber Loop Resonator
}

\author{
Yangzi Zheng, Xinyong Dong, Chunliu Zhao, Yi Li, Liyang Shao, and Shangzhong Jin
}

Institute of Optoelectronic Technology, China Jiliang University, Hangzhou 310018, China

Correspondence should be addressed to Xinyong Dong; dong_x_y@hotmail.com

Received 28 May 2013; Accepted 4 August 2013

Academic Editor: Sulaiman Wadi Harun

Copyright (C) 2013 Yangzi Zheng et al. This is an open access article distributed under the Creative Commons Attribution License, which permits unrestricted use, distribution, and reproduction in any medium, provided the original work is properly cited.

\begin{abstract}
A novel relative humidity $(\mathrm{RH})$ sensor based on a microfiber loop resonator (MLR) is proposed and experimentally demonstrated. As refractive index of the microfiber in the MLR is modified by environmental humidity, resonant wavelength of the MLR changes with $\mathrm{RH}$ level. By detecting this wavelength shift, $\mathrm{RH}$ measurement is realized with a linear response sensitivity of $1.8 \mathrm{pm} / \% \mathrm{RH}$. The obvious advantage of this technique over others is that no coating of humidity-sensitive material is required.
\end{abstract}

\section{Introduction}

Optical fiber-based relative humidity (RH) sensors have been widely studied due to their advantages such as feasibility of long-distance sensing, real-time monitoring, and immunity to electromagnetic interference. Various techniques including tapered optical fiber, heterocore optical fiber, and fiber grating have been reported previously [1-4]. Most of them contain a certain kind of RH-sensitive materials to be coated out of the fiber surface. But it brings some disadvantages, such as increasing manufacturing difficulty and reducing the measurement speed.

Recently, micro/nanofibers (MNFs), drawn from standard optical fibers have attracted a lot of attention. Due to their subwavelength diameter, MNFs leave a large fraction of the guided field outside the fiber as evanescent waves. So silica optical MNFs are promising to develop kinds of micro- and nanophotonic components and devices [5-13]. In this work, we proposed and experimentally demonstrated a novel coating-free RH fiber sensor by using a microfiber loop resonator (MLR). The microfiber is only several micrometers in diameter and there is no additional humidity-sensitive material coated on the fiber surface. The proposed sensor is therefore compact in size, fast in response, and low in cost.

\section{Sensor Configuration}

Figure 1 shows the schematic diagram of the proposed sensor. The microfiber was drawn from a standard telecom singlemode fiber (SMF-28, Corning Inc.) using a fusion splicer
(Fujikura ARC Master FSM-100P). Under the special function mode a two-step fiber-tapering program was created. In step 1, the discharge current was set as SP-45 bit and the diameter of the fiber was then tapered from $125 \mu \mathrm{m}$ to about $7 \mu \mathrm{m}$. In step 2, the discharge current was set as SP-220 bit to increase length of the $7 \mu \mathrm{m}$ fiber region to $6 \mathrm{~mm}$.

Controlling the shape of the microfiber with two translation stages under an optical microscope, we managed to coil the microfiber into a self-touching loop. The parallel alignment of the adjacent microfiber segments were achieved with the help of the surface attraction forces (Van der Waals and electrostatic), which kept both the microfiber segments together (as shown in Figure 1), apparently overcoming the elastic force that would tent to straighten out the microfiber. The two ends of the microfiber connected to the single mode fiber were used as input and output ports, respectively. We used a broadband light source (BBS) as the input source and an optical spectrum analyzer (OSA) to measure the transmission spectrum of the so-formed MLR, as shown in Figure 2. Relative humidity-sensing experiment was carried out by placing the MLR in a sealed chamber that contains two stages to fix microfiber.

\section{Experimental Results and Discussion}

After tuning the extinction ratio of the resonance spectrum by carefully adjusting the coupling region of the ring resonator, we obtained the spectral transmission curve as shown in Figure 3. In order to reduce the influence of vibration 


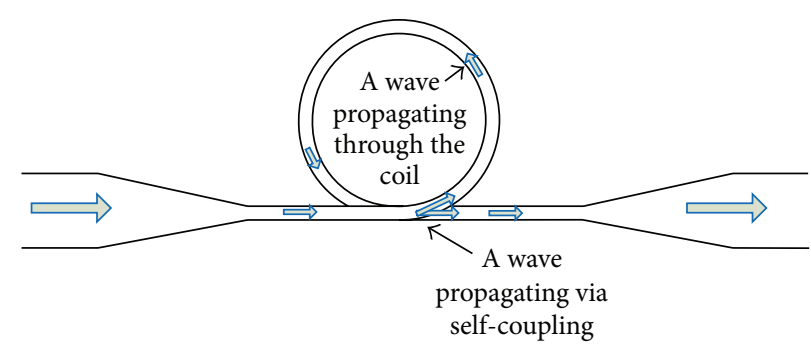

FIGURE 1: Schematic diagram of the microfiber loop resonator.

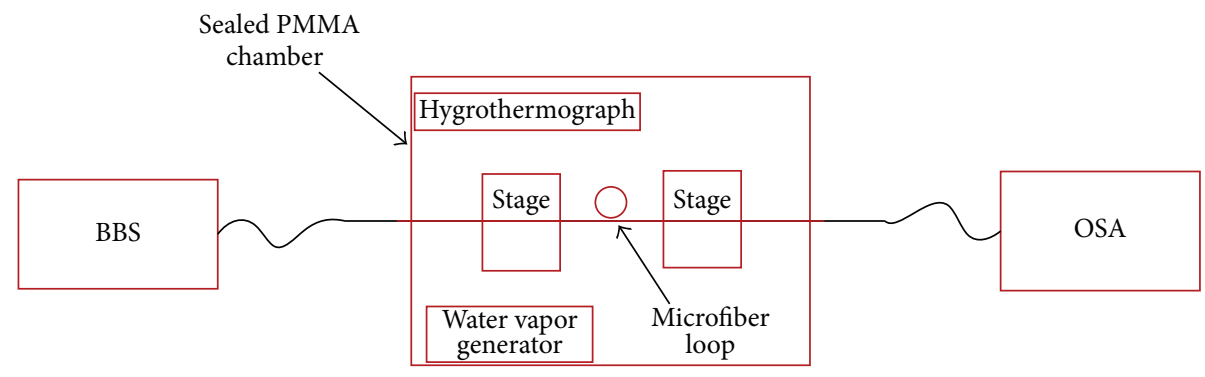

FIGURE 2: The relative humidity measurement system. BBS: broadband light source: OSA, optical spectral analyzer.

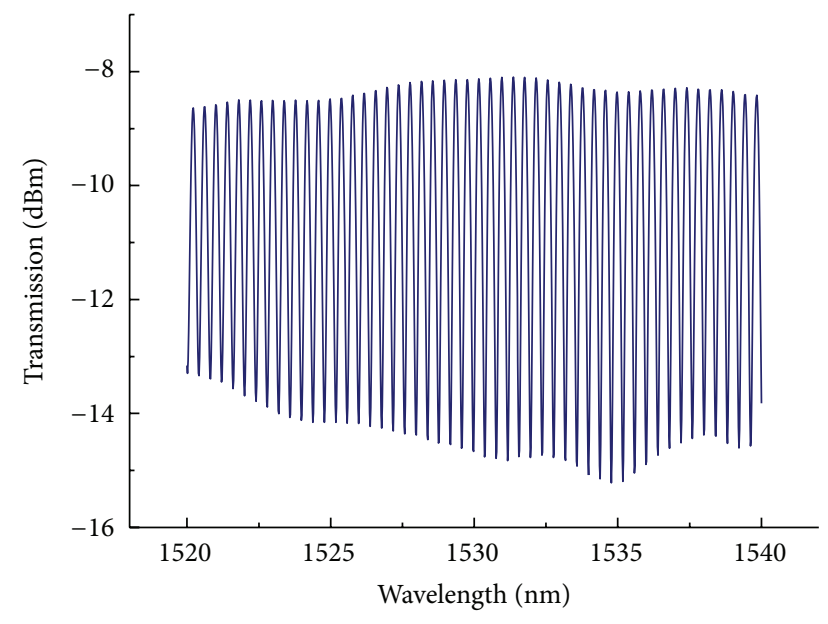

FIGURE 3: Measured transmission spectrum of the MLR.

caused by air-flow, we used a water vapor generator to change the relative humidity in the testing chamber. And the relative humidity in the chamber was monitored by using a hygrothermograph. Humidity was turned in the range between $51 \% \mathrm{RH}$ and $78 \% \mathrm{RH}$ under fixed temperature of $21.9^{\circ} \mathrm{C}$. Without dry air pumping into the chamber to decrease the relative humidity, we had to start the test under the lab relativity humidity around $50 \% \mathrm{RH}$.

When humidity level was changed, transmission spectrum of the MLR was recorded by using the OSA. Figure 4 shows several of measured spectra under different RH levels. It can be seen that resonant wavelengths of the MLR redshifted together with an increase in intensity and a slight reduction in extinction ratio when the level of relative humidity increased. Figure 5 shows the linearity between humidity and resonant wavelength shift and the square

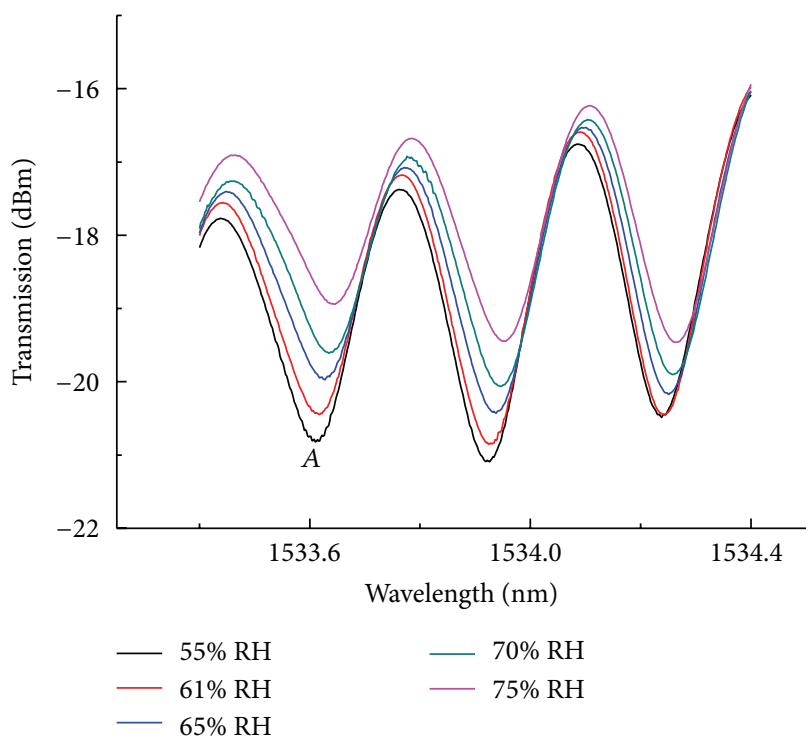

FIGURE 4: Transmission spectra of the MLR under various RH levels.

regression coefficient $\left(R^{2}\right)$ is 0.9901 . We can find that the response is in a linear relationship and the achieved sensitivity is $1.8 \mathrm{pm} / \%$ RH. Figure 6 shows the relationship between $\mathrm{RH}$ and extinction ratio/intensity of resonant wavelength $A$. The extinction ratio of resonant wavelength $A$ reduced from $3.7 \mathrm{~dB}$ to $2.1 \mathrm{~dB}$, the achieved sensitivity is $-0.064 \mathrm{~dB} / \%$ $\mathrm{RH}$, and the square regression coefficient $\left(R^{2}\right)$ is 0.9864 . Alternatively, the intensity increased from $-21.3 \mathrm{dBm}$ to $-18.5 \mathrm{dBm}$, achieved sensitivity is $0.095 \mathrm{dBm} / \% \mathrm{RH}$, and the square regression coefficient $\left(R^{2}\right)$ is 0.9895 . An increase in intensity and a slight reduction in extinction ratio may be caused by aggregation of water molecules and formation of clusters on the MLR [11, 13]. 


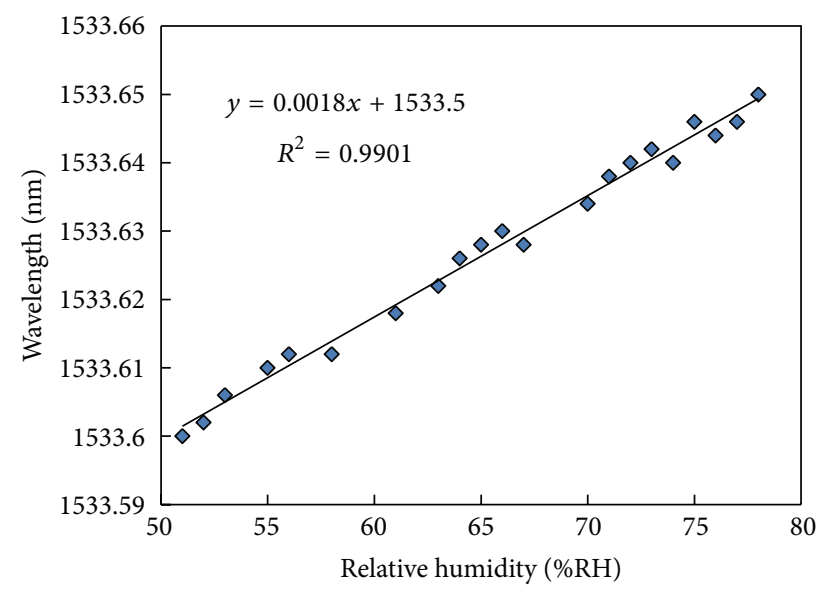

FIgURE 5: Relationship between RH and resonant wavelength $A$.

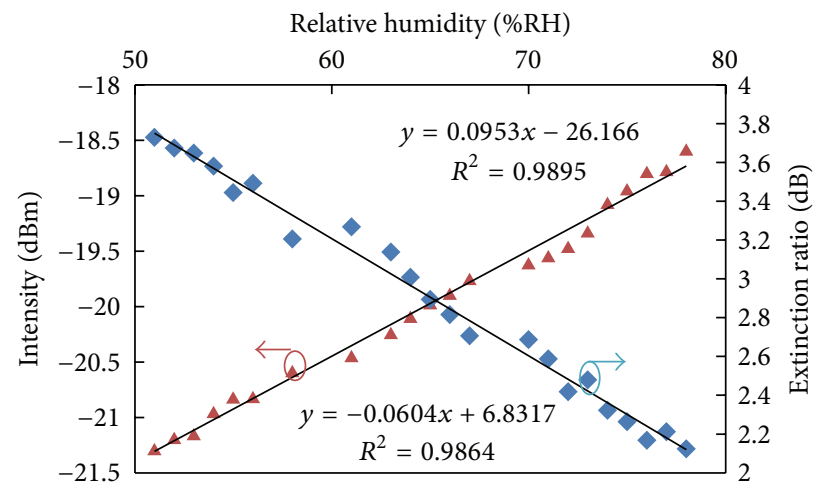

FIGURE 6: Relationship between RH and extinction ratio/intensity of resonant wavelength $A$.

The response of the MLR to humidity can be explained as follows. The porous matrix of the silicon trapped water molecules on its interior surface. These trapped water molecules increased the average density of the silicon and resulted in an increase of the refractive index [11]. Finally, resonant wavelengths of the MLR shifted with RH level as a result of the refractive index change. The MLR is also sensitive to temperature and sensitivity of $0.043 \mathrm{~dB} /{ }^{\circ} \mathrm{C}$ has been reported [14]. We plan to use another MLR that have the same parameter to monitor the influence of the temperature. Further study involving temperature compensation technique for the proposed MLR sensor is in process. Although the sensitivity of this MLR-based humidity sensor is relatively low, it can be improved by further reducing diameter of the microfiber and the obvious advantage is that the sensing element is formed by bare microfiber, no additional materials coated on its surface. It also provides potentials of fast response, low cost, and compact size.

\section{Conclusions}

A RH sensor based on an MLR without coating of any humidity sensitive material has been proposed and experimentally demonstrated. Experimental results show that linear response with sensitivity of $1.8 \mathrm{pm} / \% \mathrm{RH}$ has been achieved. Although the sensitivity is relatively low, the proposed MLRbased RH sensor shows potentials of compact size, fast response, and low cost.

\section{Conflict of Interests}

The authors of this paper do not have a direct financial relation with the commercial identities mentioned herein.

\section{Acknowledgments}

This work was supported by the National Basic Research Program of China (973 Program), under Grant no. 2010CB327804, National Natural Science Foundation of China under Grants nos. 61007050 and 61007051, and National Natural Science Foundation of Zhejiang Province, China, under Grant no. Z13F050003.

\section{References}

[1] L. Zhang, F. Gu, J. Lou, X. Yin, and L. Tong, "Fast detection of humidity with a subwavelength-diameter fiber taper coated with gelatin film," Optics Express, vol. 16, no. 17, pp. 13349-13353, 2008.

[2] X. Dong, T. Li, Y. Liu, Y. Li, C. Zhao, and C. Chan, "Polyvinyl alcohol-coated hybrid fiber grating for relative humidity sensing," Journal of Biomedical Optics, vol. 16, no. 7, Article ID 077001, 2011.

[3] Q. Wu, Y. Semenova, J. Mathew, P. Wang, and G. Farrell, "Humidity sensor based on a single-mode hetero-core fiber structure," Optics Letters, vol. 36, no. 10, pp. 1752-1754, 2011.

[4] T. Li, X. Dong, C. Chan, C. Zhao, and P. Zu, "Humidity sensor based on a multimode-fiber taper coated with polyvinyl alcohol interacting with a fiber bragg grating," IEEE Sensors Journal, vol. 12, no. 6, pp. 2205-2208, 2012.

[5] L. Tong, F. Zi, X. Guo, and J. Lou, "Optical microfibers and nanofibers: a tutorial," Optics Communications, vol. 285, pp. 4641-4647, 2012.

[6] G. Brambilla, F. Xu, P. Horak et al., "Optical fiber nanowires and microwires: fabrication and applications," Advances in Optics and Photonics, vol. 1, no. 1, pp. 107-161, 2009.

[7] G. Brambilla, "Optical fibre microwires: some "practical" devices," in Proceedings of the 24th Annual Meeting on IEEE Photonic Society (PHO '11), pp. 791-792, October 2011.

[8] M. Sumetsky, Y. Dulashko, J. M. Fini, A. Hale, and D. J. DiGiovanni, "The microfiber loop resonator: theory, experiment, and application," Journal of Lightwave Technology, vol. 24, no. 1, pp. 242-250, 2006.

[9] X. Guo and L. Tong, "Supported microfiber loops for optical sensing," Optics Express, vol. 16, no. 19, pp. 14429-14434, 2008.

[10] L. Shi, Y. Xu, W. Tan, and X. Chen, "Simulation of optical microfiber loop resonators for ambient refractive index sensing," Sensors, vol. 7, no. 5, pp. 689-696, 2007.

[11] Y. Wu, T. Zhang, Y. Rao, and Y. Gong, "Miniature interferometric humidity sensors based on silica/polymer microfiber knot resonators," Sensors and Actuators B, vol. 155, no. 1, pp. 258-263, 2011.

[12] L. Zhang, J. Lou, and L. Tong, "Micro/nanofiber optical sensors," Photonic Sensors, vol. 1, no. 1, pp. 31-42, 2011. 
[13] L. Zhang, F. Gu, J. Lou, X. Yin, and L. Tong, "Fast detection of humidity with a subwavelength-diameter fiber taper coated with gelatin film," Optics Express, vol. 16, no. 17, pp. 13349-13353, 2008.

[14] S. W. Harun, K. S. Lim, S. S. A. Damanhuri, and H. Ahmad, "Microfiber loop resonator based temperature sensor," Journal of the European Optical Society, vol. 6, Article ID 11026, 2011. 

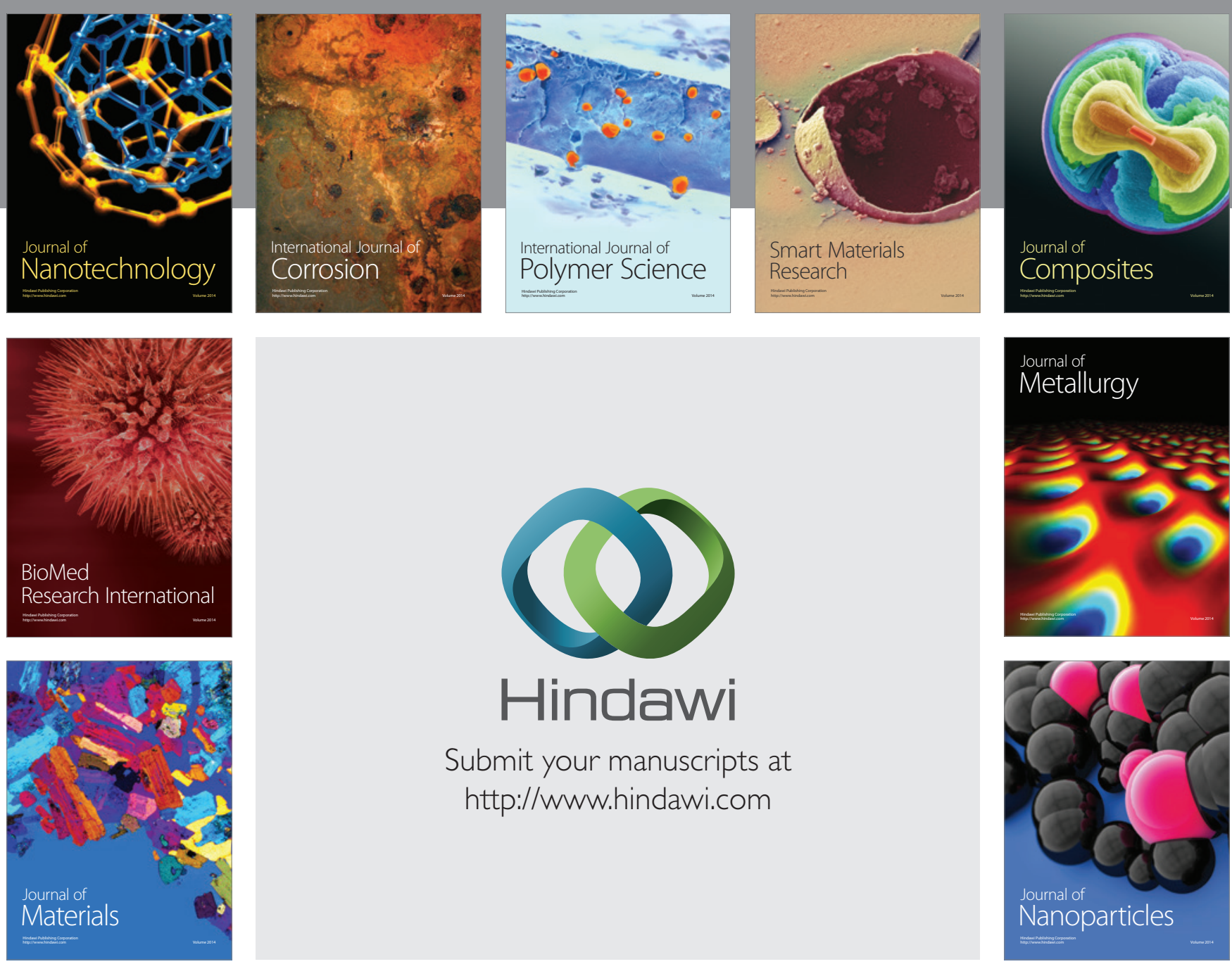

Submit your manuscripts at http://www.hindawi.com
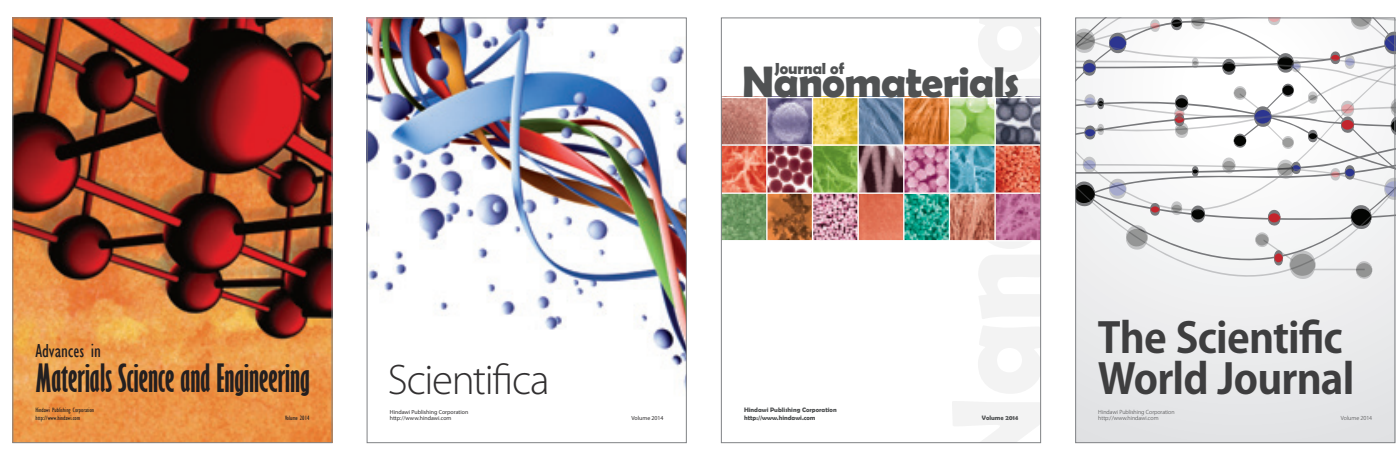

\section{The Scientific World Journal}
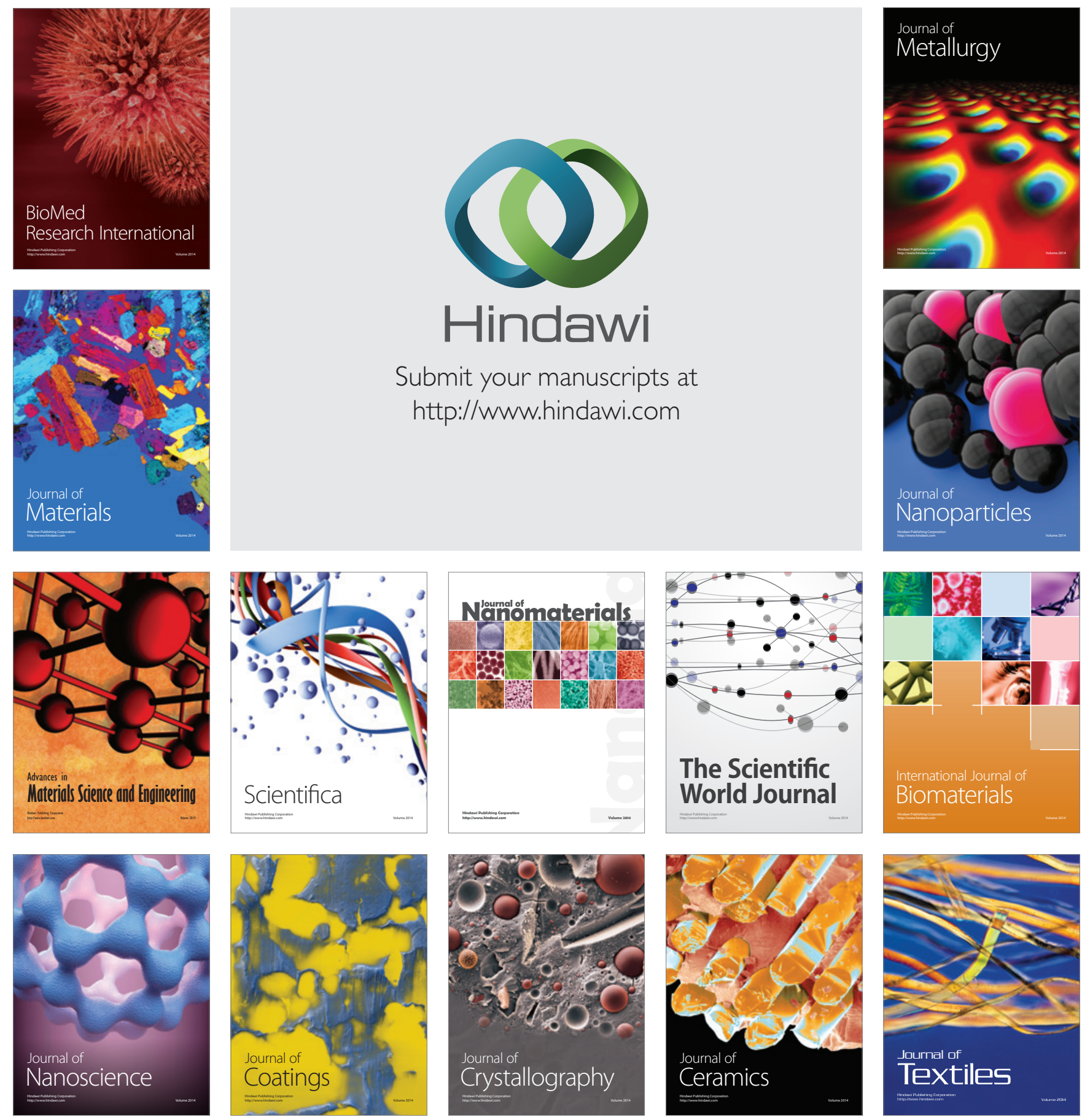